OPEN ACCESS

Edited by: Jose Antonio Lopez-Escamez, Granada University Hospital, Spain

Reviewed by: Eduardo Martin-Sanz, Hospital de Getafe, Spain

Raquel Manrique-Huarte, University of Navarra Clinic, Spain

*Correspondence: R. van de Berg raymond.vande.berg@mumc.nl

Specialty section: This article was submitted to Neuro-otology, a section of the journal Frontiers in Neurology

Received: 24 August 2016 Accepted: 18 October 2016 Published: 07 November 2016

Citation:

van den Burg EL, van Hoof M, Postma AA, Janssen AML,

Stokroos RJ, Kingma $\mathrm{H}$ and van de Berg R (2016) An Exploratory Study to Detect Ménière's Disease in

Conventional MRI Scans Using Radiomics.

Front. Neurol. 7:190.

doi: 10.3389/fneur.2016.00190

\section{An Exploratory Study to Detect Ménière's Disease in Conventional MRI Scans Using Radiomics}

\author{
E. L. van den Burg', M. van Hoof', A. A. Postma' ${ }^{2}$, A. M. L. Janssen ${ }^{3}$, R. J. Stokroos', \\ H. Kingma ${ }^{1,4}$ and R. van de Berg ${ }^{1,4 *}$ \\ ${ }^{1}$ Department of Otorhinolaryngology and Head and Neck Surgery, Maastricht University Medical Center, Maastricht, \\ Netherlands, ${ }^{2}$ Department of Radiology, Maastricht University Medical Center, Maastricht, Netherlands, ${ }^{3}$ Department of \\ Methodology and Statistics, School for Public Health and Primary Care (CAPHRI), Maastricht University, Maastricht, \\ Netherlands, ${ }^{4}$ Faculty of Physics, Tomsk State University, Tomsk, Russian Federation
}

Objective: The purpose of this exploratory study was to investigate whether a quantitative image analysis of the labyrinth in conventional magnetic resonance imaging (MRI) scans using a radiomics approach showed differences between patients with Ménière's disease (MD) and the control group.

Materials and methods: In this retrospective study, MRI scans of the affected labyrinths of 24 patients with MD were compared to the MRI scans of labyrinths of 29 patients with an idiopathic asymmetrical sensorineural hearing loss. The 1.5- and 3-T MRI scans had been previously made in a clinical setting between 2008 and 2015. 3D Slicer 4.4 was used to extract several substructures of the labyrinth. A quantitative analysis of the normalized radiomic image features was performed in Mathematica 10. The image features of the two groups were statistically compared.

Results: For numerous image features, there was a statistically significant difference ( $p$-value $<0.05$ ) between the MD group and the control group. The statistically significant differences in image features were localized in all the substructures of the labyrinth: 43 in the anterior semicircular canal, 10 in the vestibule, 22 in the cochlea, 12 in the posterior semicircular canal, 24 in the horizontal semicircular canal, 11 in the common crus, and 44 in the volume containing the reuniting duct. Furthermore, some figures contain vertical or horizontal bands (three or more statistically significant image features in the same image feature). Several bands were seen: 9 bands in the anterior semicircular canal, 1 band in the vestibule, 3 bands in the cochlea, 0 bands in the posterior semicircular canal, 5 bands in the horizontal semicircular canal, 3 bands in the common crus, and 10 bands in the volume containing the reuniting duct.

Conclusion: In this exploratory study, several differences were found in image features between the MD group and the control group by using a quantitative radiomics approach on high resolution T2-weighted MRI scans of the labyrinth. Further research should be aimed at validating these results and translating them in a potential clinical diagnostic method to detect MD in MRI scans.

Keywords: Ménière's disease, MRI, imaging, radiomics, quantitative image analysis, labyrinth, 3D models, vertigo 


\section{INTRODUCTION}

Ménière's disease (MD) is a disorder of the inner ear, which is characterized by recurrent attacks of vertigo. These attacks are accompanied by a fluctuating sensorineural hearing loss and tinnitus or a sense of fullness in the affected ear (1). The exact cause and pathophysiology of MD is unclear and includes genetic, anatomic, metabolic, endocrine, autoimmune, vascular, allergic, viral, and traumatic factors (2). Histopathologic examination in patients with MD shows a distention of Reissner's membrane (endolymphatic hydrops) in the cochlea or endolymphatic compartment of the labyrinth $(3,4)$. Since histopathologic examination (the gold standard) is not possible in a clinical setting, the diagnosis depends on the symptoms of $\operatorname{MD}(1,5)$. For the diagnosis of definite MD, one of the criteria is that other causes of the symptoms have been excluded. Magnetic resonance imaging (MRI) is often indicated because of asymmetrical sensorineural hearing loss and to exclude other possible causes (6). However, it remains difficult to differentiate between MD and other causes of vertigo. Therefore, new imaging techniques are under investigation as a MD diagnostic, which include cone beam computed tomography (7) and MRI enhanced by invasive contrast agents such as gadolinium $(8,9)$. The administration of an intratympanic gadolinium injection is an invasive procedure and although adverse events are rare after intravenous contrast media, they are known to occur (10). A non-invasive imaging technique would be preferable as it could be argued that the invasiveness of this procedure does not justify the (potential) gain in diagnostic information it provides.

Here, an alternative approach is taken by analyzing quantitative data in conventional MRI scans. The evidence is increasing that with new imaging processing and analysis techniques, the evidence is increasing that with new imaging processing and analysis techniques, more information can be gathered from standard imaging modalities $(11,12)$. Texture analysis uses features such as the distribution of gray levels in an area or volume in an MRI scan (13). Radiomics refers to the extraction and analysis of such quantitative image features from medical images obtained with computed tomography (CT), positron emission tomography (PET), or MRI (14). These quantitative image features provide additional information about the analyzed structures that are not necessarily perceptually visible by the (neuro)radiologist. Another advantage is that standard-of-care images from the clinic can be used (14). In the process of radiomics, there are several steps to be followed (15). The first step is the acquisition of imaging (preferably standard-of-care). Second, an anatomical region has to be segmented to define the region of interest on the acquired image volume. In the study, here, this is the labyrinth. Third, quantitative image features have to be calculated from this segmentation (such as the mean of the intensities in the segmentation). Finally, these quantitative image features can be used for statistical analysis. Several recent studies have shown that radiomics can be used to obtain information about, for example, tumor phenotypes and prognosis $(11,12)$, tumor biomarkers (16), and distant metastasis (17). Studies have also shown structural differences in patients with amyotrophic lateral sclerosis (13) and Alzheimer's disease (18) when compared to healthy subjects.
The purpose of this exploratory study was to investigate whether a quantitative image analysis of the labyrinth in conventional MRI scans using a radiomics approach showed differences between patients with MD and the control group.

\section{MATERIALS AND METHODS}

\section{Ethical Considerations}

This study was performed in accordance with the guidelines outlined by Dutch legislation. According to the Medical Research Involving Human Subjects Act (WMO), ethical approval was not required due to the retrospective nature and anonymization of the data.

\section{Study Population}

A retrospective study was performed. Patients with MD were identified from the patient registration system from a tertiary center. Patients with definite MD according to the criteria as accepted by the American Academy of Otolaryngology-Head and Neck Surgery (AAO-HNS) (1) were included, since these were the most recent criteria for $\mathrm{MD}$ during the inclusion period. In retrospect, the diagnostic criteria for definite $\mathrm{MD}$ as formulated in 2015 by the Bárány Society (5) also apply to all the patients with MD included in this study. Furthermore, an MRI scan of the cerebellopontine angle made in two preselected MRI scanners was required for inclusion. Since this retrospective study was performed in a tertiary center, many patients with MD had an MRI scan made in another center and could therefore not be included in this study. The labyrinth affected by MD was used in the study. In case of bilateral MD, one of the labyrinths was chosen. The exclusion criterion was motion artifacts on the MRI scan (the criterion was that the inner ear should be sharply delineated). The control group consisted of patients with idiopathic asymmetrical sensorineural hearing loss. These patients were chosen as controls, since this was a retrospective study and no MRI scans from people without hearing loss were available. The labyrinth least affected by hearing loss was included in the study, because this labyrinth was considered to be most representative for a healthy person. In the clinical setting, conventional clinical tests at the discretion of the ENT specialists were performed to diagnose the patients with an idiopathic asymmetrical sensorineural hearing loss. The exclusion criteria were composed of a documented history of vertigo or balance disorders and having motion artifacts on the MRI scan. The control group was matched to the MD group by scanning date to minimize biases which might arise from differences in scanning protocols over the years.

\section{MR Imaging}

A Philips Intera MRI scanner (1.5 T) and a Philips Achieva MRI scanner $(3 \mathrm{~T})$ had been used to obtain the 3D High resolution T2-weighted images (Philips Nederland B.V., Eindhoven, The Netherlands). The scans had been previously made in a clinical setting between 2008 and 2015. The scan parameters were not constant, as can be seen in Table 1. All the scans had been previously evaluated by a radiologist. 
TABLE 1 | Scan parameters.

\begin{tabular}{|c|c|c|c|}
\hline \multirow[b]{2}{*}{ Scanning date } & \multirow{2}{*}{$\begin{array}{l}\text { 1.5-T MRI scanner } \\
\text { 01-08-2012 until } \\
17-05-2015\end{array}$} & \multicolumn{2}{|c|}{ 3-T MRI scanner } \\
\hline & & $\begin{array}{l}28-05-2008 \text { until } \\
31-05-2011\end{array}$ & $\begin{array}{l}06-02-2013 \text { until } \\
26-04-2015\end{array}$ \\
\hline Repetition time (ms) & 1500 & 2000 & 1500 \\
\hline Echo time (ms) & $\begin{array}{l}\text { Between } 169 \\
\text { and } 182\end{array}$ & 200 & $\begin{array}{l}\text { Between } 193 \\
\text { and } 195\end{array}$ \\
\hline Slice thickness (mm) & 0.6 & 1.0 & 0.8 \\
\hline $\begin{array}{l}\text { Spacing between } \\
\text { the slices }(\mathrm{mm})\end{array}$ & 0.3 & 0.5 & 0.4 \\
\hline Echo train length & 40 & 59 & 40 \\
\hline $\begin{array}{l}\text { Magnetic field } \\
\text { strength }(T)\end{array}$ & 1.5 & 3 & 3 \\
\hline
\end{tabular}

Scan parameters and scanning data of the included MRI scans.

\section{Image Extraction of the Labyrinth}

3D Slicer 4.4, an open source software package for visualization and image analysis $(19,20)$ was used to extract the (sub)volumes from the MRI scans. First, a label was created in the shape of the labyrinth for the segmentation. The labyrinth was segmented into several substructures: the cochlea, the volume containing the reuniting duct, the vestibule, the semicircular canals, and the common crus (Figure 1). The labels were used as masks for intensity data extraction and to generate 3D models to measure the surface area and the volume of the different substructures and the labyrinth.

\section{Radiomic Feature Extraction and Statistical Analysis}

Mathematica 10 (Wolfram Research, Champaign, IL, USA) was used for the radiomic feature extraction and statistical analysis. 3D models from the separate substructures were created with the edited MRI scans. These 3D models were modified by using 25 different image processing filters, resulting in a total of 26 primary radiomic features. A few examples are the entropy filter, the Laplacian filter, and the Fourier DCT filter. Every model was used to calculate 23 secondary radiomic features, which were statistical values such as the minimal intensity, the maximal intensity, and the mean intensity. The radiomic features were based on standard functions available in Mathematica 10. A list of different functions used can be found in Tables 2 and 3. In total, 598 image features were extracted. Because of differences between MRI scanners and scanning protocols, the image features were normalized. This was done by dividing the features from the different substructures of one patient by the features of the entire labyrinth of the same patient, thereby diminishing potential systematic differences introduced by different scanner's and scanner protocols (Table 1). For the statistical analysis of the radiomic image features, a permutation test was used. A two-sided $p$-value of $<0.05$ was considered significant.

\section{RESULTS}

\section{Ménière's Disease Group and Control Group}

In total, 24 patients who met the inclusion criteria for MD were included in this study. The control group consisted of 29 patients.

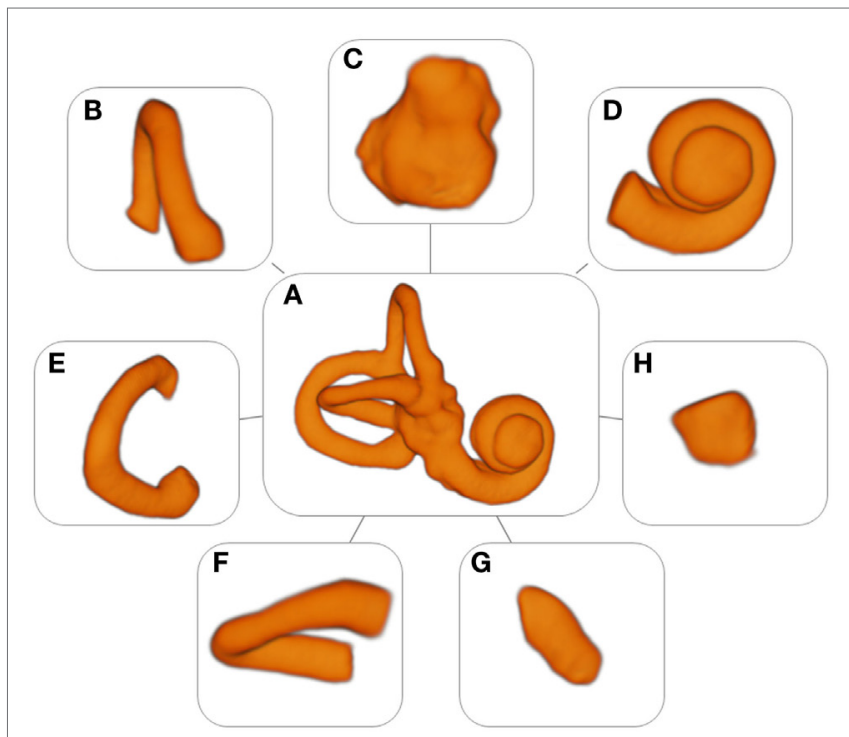

FIGURE 1 | Models of the labyrinth and its substructures. (A) Labyrinth, (B) anterior semicircular canal, (C) vestibule, (D) cochlea, (E) posterior semicircular canal, (F) horizontal semicircular canal, (G) common crus, and $\mathbf{( H )}$ volume containing the reuniting duct.

\section{TABLE 2 | Primary radiomic features.}

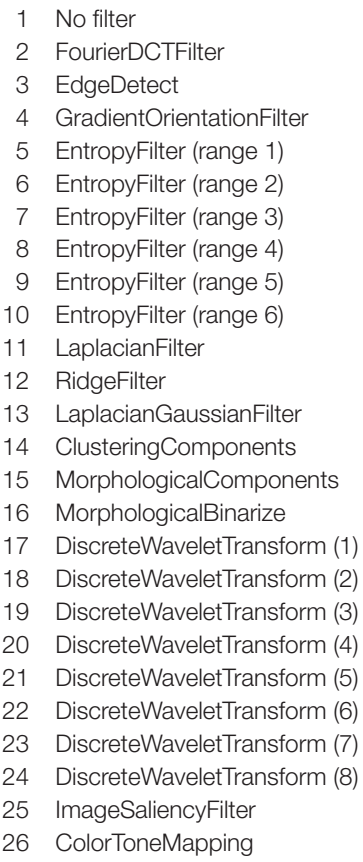

Functions used in Mathematica 10 (Wolfram Research, Champaign, IL, USA) to create the primary radiomic features.

The two groups were similar with regard to age, the labyrinth analyzed, and distribution between MRI scanners and scanning dates (Table 4). More men than women were included in both groups. The median hearing loss in the analyzed ear was higher in the MD group than in the control group. 


\section{Surface Area and Volume}

The surface area and volume of the labyrinth and its separate substructures were not significantly different between the two groups (Table 5).

\section{Models}

A set of 53 detailed models of the labyrinth and its separate substructures were formed (Figure 1). These models were used to extract the radiomic image features. In addition, they provided an accurate three-dimensional image of the labyrinth and its substructures.

\section{Radiomic Image Features}

A total of 598 radiomic image features were analyzed in every substructure of the labyrinth. In all the separate substructures,

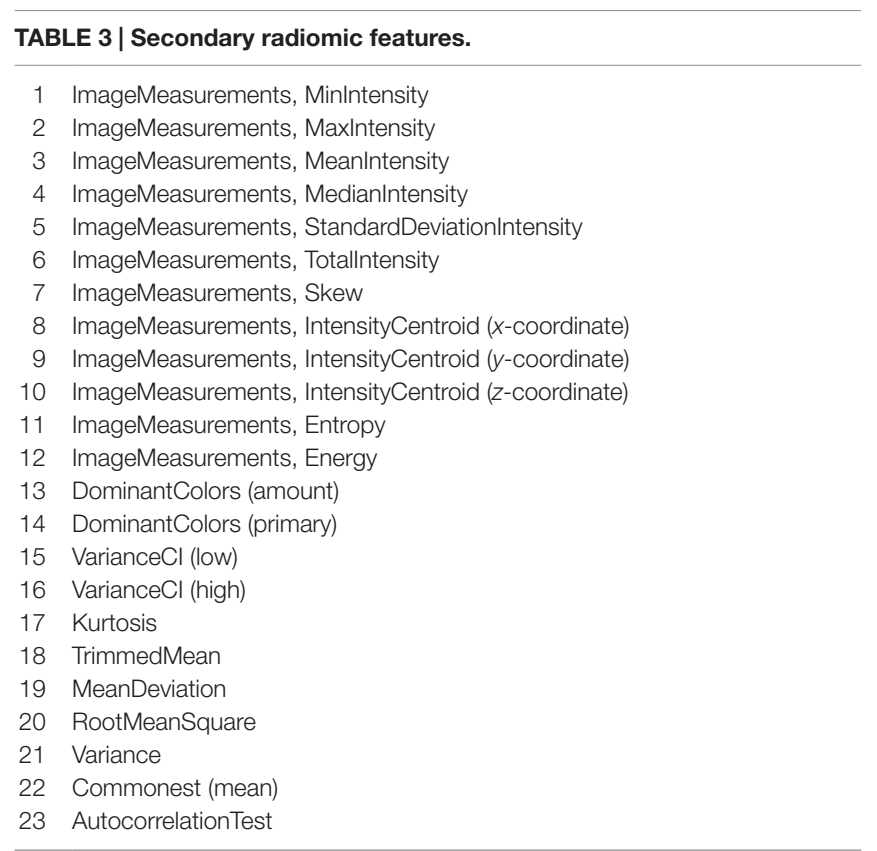

Functions used in Mathematica 10 Wolfram Research, Champaign, IL, USA) to create the secondary radiomic features. statistically significant differences between the MD group and the control group were seen in several image features (Figures 2A-G). Figure $\mathbf{2} \mathbf{H}$ shows the p-value legend. In the figures, differences are seen between the amount of statistically significant image features in the different substructures: 43 in the anterior semicircular canal, 10 in the vestibule, 22 in the cochlea, 12 in the posterior semicircular canal, 24 in the horizontal semicircular canal, 11 in the common crus, and 44 in the volume containing the reuniting duct. For example, there are more imaging features that resulted in statistically significance in the volume containing the reuniting duct and in the anterior semicircular canal than in the other substructures. Furthermore, some figures contain

TABLE 4 | Patient demographics.

\begin{tabular}{|c|c|c|c|}
\hline & & $\begin{array}{c}\text { Ménière's } \\
\text { disease group } \\
(n=24)\end{array}$ & $\begin{array}{l}\text { Control } \\
\text { group } \\
(n=29)\end{array}$ \\
\hline $\begin{array}{l}\text { Age at the moment of } \\
\text { scanning in years } \\
\text { [median (interquartile range)] }\end{array}$ & & $61(51-71)$ & 56 (48.8-64.3) \\
\hline Gender (F/M) & & $37.5 / 62.5 \%$ & $13.8 / 86.2 \%$ \\
\hline Analyzed labyrinth (left/right) & & $50.0 / 50.0 \%$ & $37.9 / 62.1 \%$ \\
\hline MRI-scanner (1.5/3 T) & & $41.7 / 58.3 \%$ & $34.5 / 65.5 \%$ \\
\hline Scanning date (range) & & $\begin{array}{c}29-10-2008 \text { until } \\
17-05-2015\end{array}$ & $\begin{array}{c}28-05-2008 \text { until } \\
26-04-2015\end{array}$ \\
\hline \multirow[t]{8}{*}{ Distribution of scanning dates } & 2008 & $4.2 \%$ & $3.5 \%$ \\
\hline & 2009 & $4.2 \%$ & $3.5 \%$ \\
\hline & 2010 & $4.2 \%$ & $3.5 \%$ \\
\hline & 2011 & $4.2 \%$ & $3.5 \%$ \\
\hline & 2012 & $12.5 \%$ & $20.7 \%$ \\
\hline & 2013 & $25.0 \%$ & $48.3 \%$ \\
\hline & 2014 & $33.3 \%$ & $10.3 \%$ \\
\hline & 2015 & $12.5 \%$ & $6.9 \%$ \\
\hline $\begin{array}{l}\text { Average hearing loss } \\
\text { in analyzed ear in } \mathrm{dB} \\
\text { [median (interquartile range)] }\end{array}$ & & $61(42-75)$ & $13(8-25.5)$ \\
\hline Bilateral MD (yes/no) & & $20.8 / 79.2 \%$ & - \\
\hline
\end{tabular}

Information about the Ménière's disease group and the control group.

aThe audiogram closest to the date of scanning was used.

TABLE 5 | Surface area and volume of the labyrinth and its separate substructures.

\begin{tabular}{|c|c|c|c|c|}
\hline & & Ménière's disease $(n=24)$ & Control group $(n=29)$ & $p$-Value \\
\hline \multirow{8}{*}{$\begin{array}{l}\text { Surface area in } \mathrm{mm}^{2} \\
\text { [median (interquartile range)] }\end{array}$} & Cochlea & 135.29 (129.08-146.86) & $144.31(135.91-150.45)$ & 0.122 \\
\hline & Volume containing the reuniting duct & 31.06 (25.64-35.14) & 27.72 (25.19-31.78) & 0.195 \\
\hline & Vestibule & 95.39 (91.97-104.12) & $95.43(89.78-107.69)$ & 0.721 \\
\hline & Anterior semicircular canal & 70.03 (63.34-74.12) & $68.82(61.25-77.39)$ & 0.514 \\
\hline & Posterior semicircular canal & $79.48(70.77-86.47)$ & 82.74 (71.33-90.04) & 0.574 \\
\hline & Horizontal semicircular canal & $61.23(50.92-68.34)$ & $58.89(50.48-65.90)$ & 0.688 \\
\hline & Common crus & $21.38(18.86-23.78)$ & 23.44 (19.85-25.82) & 0.284 \\
\hline & Labyrinth & 435.63 (412.51-478.74) & 442.97 (413.05-473.11) & 0.649 \\
\hline \multirow[t]{8}{*}{ Volume in $\mathrm{mm}^{3}$ [median (interquartile range)] } & Cochlea & $80.22(74.96-90.51)$ & 86.80 (76.04-97.29) & 0.335 \\
\hline & Volume containing the reuniting duct & $12.82(9.47-14.86)$ & $10.53(9.16-12.86)$ & 0.172 \\
\hline & Vestibule & $65.27(60.04-72.31)$ & $65.21(58.07-76.90)$ & 0.957 \\
\hline & Anterior semicircular canal & $22.73(17.65-26.32)$ & $20.71(17.00-25.10)$ & 0.437 \\
\hline & Posterior semicircular canal & 26.49 (21.43-29.49) & $27.63(20.16-31.04)$ & 0.979 \\
\hline & Horizontal semicircular canal & 20.42 (14.03-23.78) & 18.19 (13.38-21.66) & 0.469 \\
\hline & Common crus & $7.01(5.61-8.21)$ & $8.14(5.84-9.15)$ & 0.348 \\
\hline & Labyrinth & 236.26 (202.02-272.98) & 239.68 (207.96-267.81) & 0.936 \\
\hline
\end{tabular}

The surface area and volume of the labyrinth and its separate substructures. 
vertical or horizontal bands, which show that there are several image features statistically significant in the same primary or secondary image feature. We have defined a band as three or more statistically significant image features in the same row or column. For example, Figure $\mathbf{2} \mathrm{C}$ shows a vertical band in the twelfth primary radiomic feature, and Figure $\mathbf{2 A}$ shows a horizontal band in the ninth secondary radiomic feature. In Figures 2A-G, several bands are seen: 9 bands in the anterior semicircular canal, 1 band in the vestibule, 3 bands in the cochlea, 0 bands in the posterior semicircular canal, 5 bands in the horizontal semicircular canal, 3 bands in the common crus, and 10 bands in the volume containing the reuniting duct.

\section{DISCUSSION}

Significant differences in radiomics image features between the MD group and the control group were found in all the substructures of the labyrinth. The band-like patterns shown in Figures 2A-G are more important than just the significant $p$-values as its interpretation is less at risk for chance findings because no corrections for multiple testing were performed. These bands indicate that primary or secondary radiomic modifications amplify features with a certain consistency. The differences found in the substructures do not necessarily reflect visually perceptible differences. Research has been performed into the histopathology
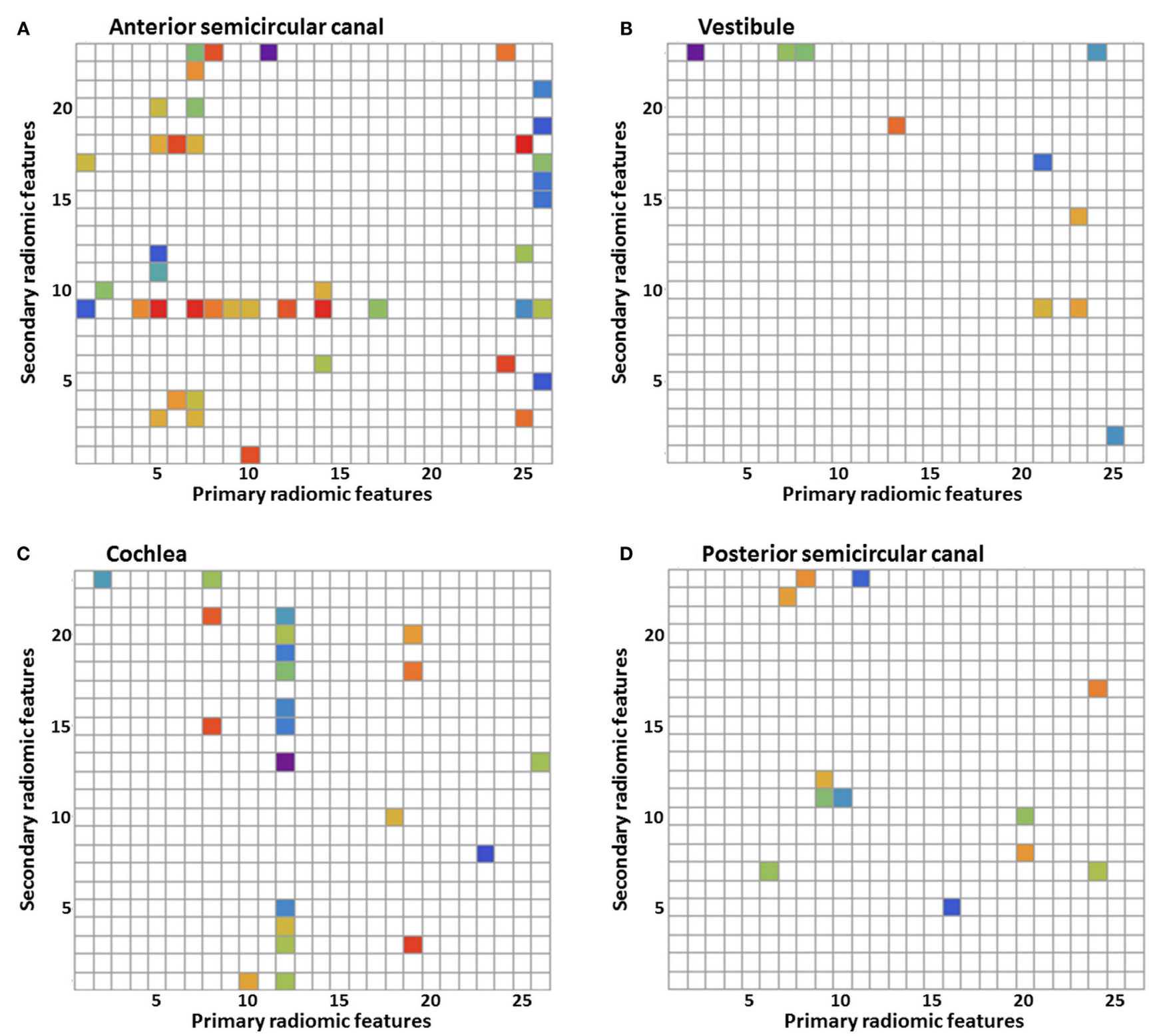

FIGURE 2 | Results of the statistical analysis of the radiomic image features 


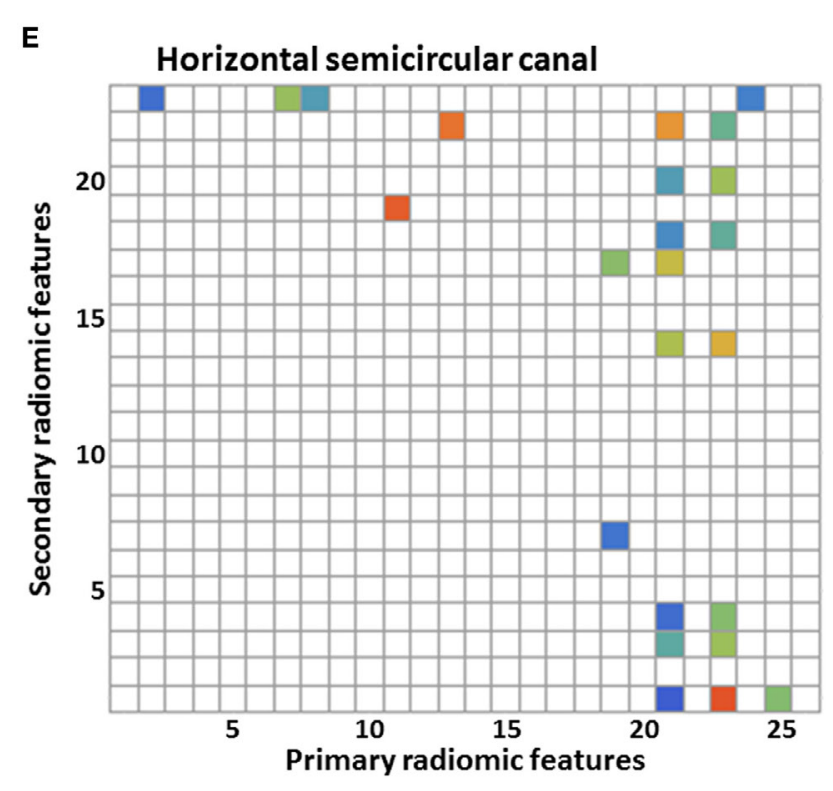

G

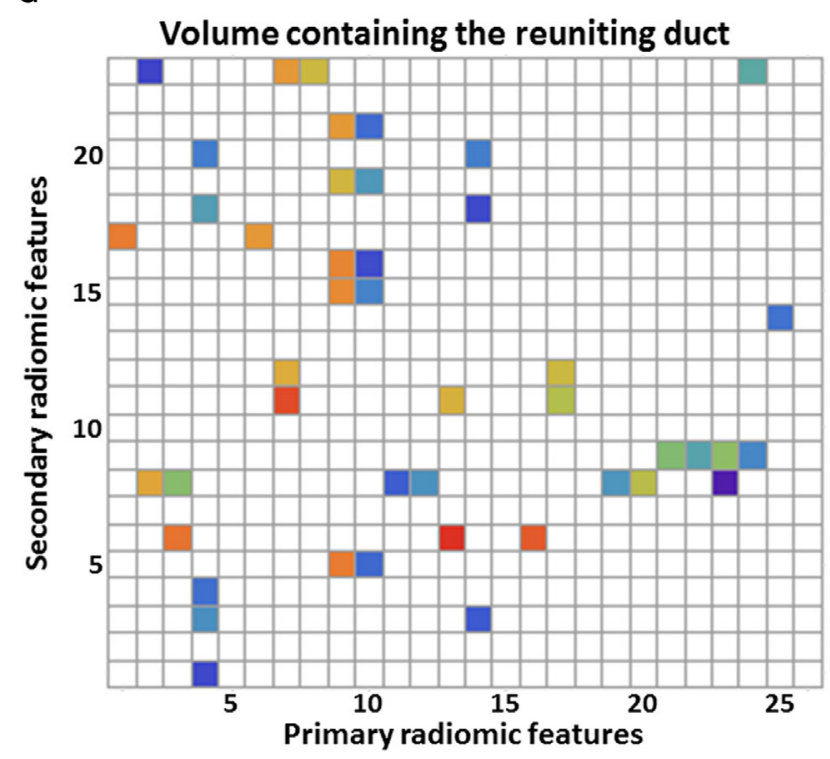

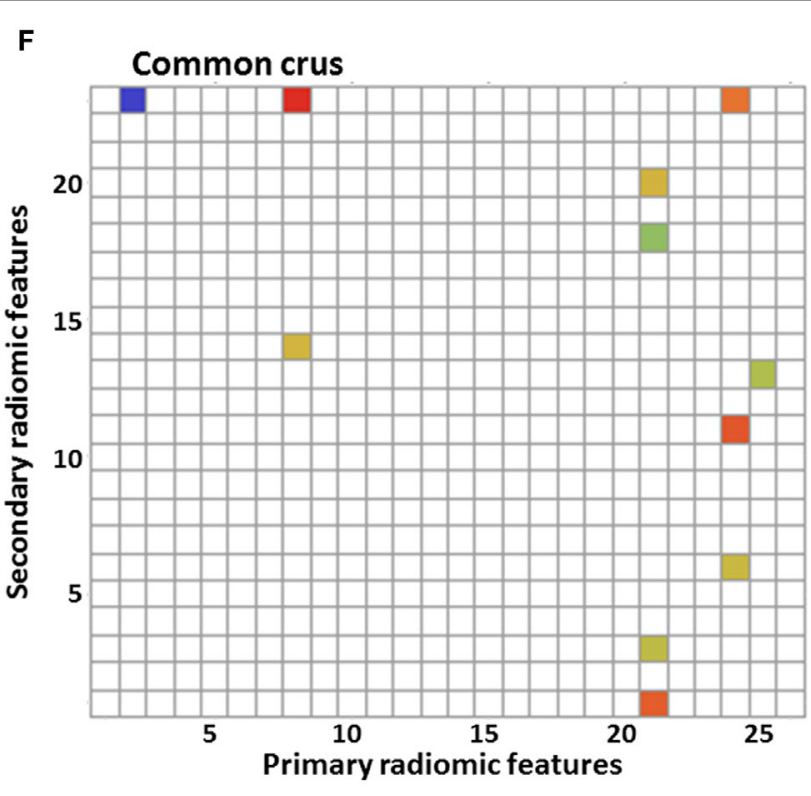

H

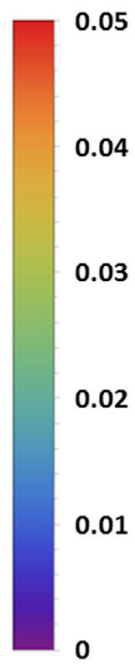

FIGURE 2 | Continued

Every $p$-value $<0.05$ is shown in color. Primary radiomic features are shown on the $x$-axis; these represent the different image processing filters. The secondary radiomic features are shown on the $y$-axis; these are the statistical values calculated from the primary radiomic features. A list of the primary radiomic features can be found in Table 2 and a list of the secondary radiomic features can be found in Table 3. A vertical band means that there are several significant image features in one primary radiomic feature, and a horizontal band means that there are several significant image features in one secondary radiomic feature. The figures represent one of the substructures of the labyrinth: (A) anterior semicircular canal, (B) vestibule, (C) cochlea, (D) posterior semicircular canal, (E) horizontal semicircular canal, (F) common crus, (G) volume containing the reuniting duct, and $\mathbf{( H )} p$-value legend.

of $\operatorname{MD}(4,21)$ also using proteomic techniques (22) and qualitative imaging techniques (7). This might provide an explanation for the observed differences in image features. The histopathology observed in a study of the temporal bones of patients with MD is endolymphatic hydrops (4). A distension, herniation, and rupture of the endolymph compartment were not necessarily confined to the cochlea. Pathologic changes were found to be present in the whole labyrinth, though the canals and common crus were affected the least (21). In another study, it was suggested that an inflammatory or autoimmune reaction in the inner ear may cause damage to the epithelial layers surrounding the endolymphatic space (22). Results found in this study correspond with these findings, since a significant difference was found in all the substructures of the labyrinth. However, a meta-analysis of temporal bone reports showed that the lesion distribution was orderly from the cochlea to the saccule, utricle, ampullae, and then the canal 
system (21). In our study, we did not find this order, since there were more statistically significant image features in, for example, the anterior semicircular canal than in the cochlea. The difference in image features could hypothetically be explained by the damage to or different distribution of differently charged fluids in several parts of the labyrinth, causing a different distribution of the intensities. A recent study using a proteomic approach found differences in protein composition of the inner ear fluid in MD patients in comparison to controls. The inner ear fluid of MD patients contained other immunoglobulins and its variants (22). Since $\gamma$-globulin has a higher relaxivity in serum (23), it could be a possible explanation for the differences in image features found in this study. Another study found a significant difference in the shape of the reuniting duct using cone beam CT (7). In this study, more imaging features that resulted in statistically significance were found in the volume containing the reuniting duct than in the other substructures, but this volume was also considerably smaller increasing the risk on a chance finding.

\section{Future Clinical Implementation}

The next step in exploring the application of radiomics for diagnosing patients with MD is to determine the accuracy as a diagnostic tool. For this purpose, an extensive internal and external validation is necessary. Recent research demonstrated that machine learning and radiomics can be used to predict overall survival in lung cancer patients (24). To investigate whether machine learning could be used for diagnosing MD patients, it should be trained and validated on two separate sets of data. The use of an independent test set would provide information on the diagnostic accuracy of this method. The advantage of using machine learning in combination with radiomics is that the analysis of the labyrinth could possibly be done autonomously as automatic image segmentation and registration are already feasible (25). The scalability that results from such a setup could possibly reshape diagnostics of the inner ear in the field of (neuro) radiology. In addition, it might be possible to identify patients who for some reason are difficult to diagnose by using the criteria for definite $\mathrm{MD}$, for example, because of fluctuating hearing loss.

These results are promising, but radiomics cannot be used in the diagnosis of patients with MD yet. MD shows several gradations that are not yet distinguished objectively but possibly could be in the future by performing a cluster analysis on image features. Also in clinical practice, it is necessary to differentiate between three populations: patients with $\mathrm{MD}$, patients with other balance disorders, and patients with complaints of dizziness which cannot be objectified (yet) (Figure 3). To enhance the differentiation between these groups, it could be helpful to cluster the relevant features to form a more robust model. Furthermore, during the last several years, several studies have been performed into the visualization of endolymphatic hydrops by using intravenous or intratympanic contrast agents such as gadolinium $(8,9,26)$. Studies have been performed that have proposed a grading system for endolymphatic hydrops on MRI $(27,28)$ or that found a correlation between the progression of disease and endolymphatic hydrops on MRI (29). Further research is necessary to compare the radiomics method to the

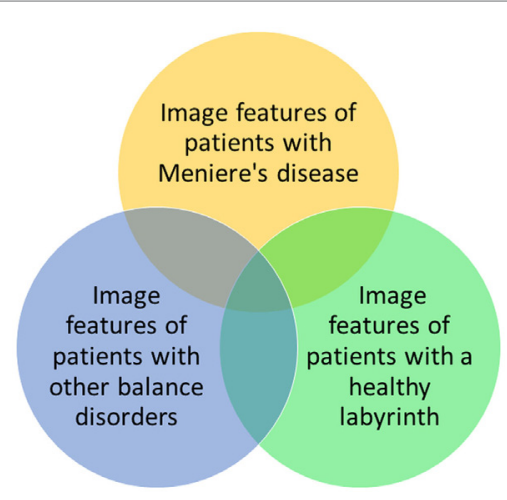

FIGURE 3 | Possible relationships between image features of patients with Ménière's disease, patients with other balance disorders, and patients with a healthy labyrinth. In this study, the yellow and green groups are investigated.

use of MRI enhanced by contrast agents, to see if similar results are found. By clustering several image features, the feasibility of a grading system in the radiomics method or a correlation between the progression of disease or progression of hearing loss can also be explored. The clustering of features can possibly also be used to separate hitherto unidentified subtypes of MD. Another advantage of the radiomics method is that no invasive contrast agents are necessary.

\section{Limitations}

A limitation in this study is that it did not include other balance disorders or other otologic conditions that could alter the composition of the labyrinth. The aim of this exploratory study instead was to investigate whether radiomic image features allow for differences to be detected between patients with MD and controls on conventional MRI scans and to provide a proof of concept for this method. Furthermore, since the aim was not to identify a single feature that could be used to diagnose a patient with MD but to identify features that could in the future be combined and used for subsequent machine learning, no correction for multiple testing was performed. Doing so would minimize false positives but could also obscure potentially important features. The statistical significant results could therefore include false positives.

Despite having two similar groups in terms of demographics (Table 4) and surface area and volume of the substructures (Table 5), confounding differences concerning the MRI scanner setup cannot be completely ruled out in this relatively small sample size (Table 1). To minimize the effect of different scan parameters, the control group was matched to the MD group by scanning date. Furthermore, by normalizing the values, it was deemed possible to compare the data, because relative differences could be analyzed. On the other hand, the variability in MRI scanners in this study might also be an advantage. If radiomics could be used clinically for the diagnosis of MD in the future, it could be more widely implemented if the method is robust and independent of the type of MRI scanner or scanning 
parameters. Furthermore, we have included both unilateral and bilateral MD patients in this study, seeing that in the future the model would be more robust if both types of MD were included. Even though the etiopathogenesis might not be the same, the structural differences in the labyrinth are thought to be similar (21) and both show endolymhatic hydrops on MRI enhanced by contrast agents (30).

\section{CONCLUSION}

In this exploratory study, several differences were found in image features between the MD group and the control group by using a quantitative radiomics approach on high resolution T2-weighted MRI scans of the labyrinth. Further research should be aimed at

\section{REFERENCES}

1. Committee on Hearing and Equilibrium guidelines for the diagnosis and evaluation of therapy in Meniere's disease. Otolaryngol Head Neck Surg (1995) 113:181-5. doi:10.1016/S0194-5998(95)70102-8

2. Chiarella G, Petrolo C, Cassandro E. The genetics of Meniere's disease. Appl Clin Genet (2015) 8:9-17. doi:10.2147/tacg.s59024

3. Hallpike CS, Cairns H. Observations on the pathology of Meniere's syndrome. J Laryngol Otol (1938) 53:625-55. doi:10.1017/S0022215100003947

4. Foster CA, Breeze RE. Endolymphatic hydrops in Meniere's disease: cause, consequence, or epiphenomenon? Otol Neurotol (2013) 34:1210-4. doi:10.1097/MAO.0b013e31829e83df

5. Lopez-Escamez JA, Carey J, Chung W-H, Goebel JA, Magnusson M, Mandalà M, et al. Diagnostic criteria for Menière's disease. J Vestib Res (2015) 25:1-7. doi:10.3233/VES-150549

6. Harcourt J, Barraclough K, Bronstein AM. Meniere's disease. BMJ (2014) 349:g6544. doi:10.1136/bmj.g6544

7. Yamane H, Iguchi H, Konishi K, Sakamaoto H, Wada T, Fujioka T, et al. Three-dimensional cone beam computed tomography imaging of the membranous labyrinth in patients with Meniere's disease. Acta Otolaryngol (2014) 134:1016-21. doi:10.3109/00016489.2014.913315

8. Nakashima T, Naganawa S, Sugiura M, Teranishi M, Sone M, Hayashi H, et al. Visualization of endolymphatic hydrops in patients with Meniere's disease. Laryngoscope (2007) 117:415-20. doi:10.1097/MLG.0b013e31802c300c

9. Nakashima T, Naganawa S, Teranishi M, Tagaya M, Nakata S, Sone M, et al. Endolymphatic hydrops revealed by intravenous gadolinium injection in patients with Meniere's disease. Acta Otolaryngol (2010) 130:338-43. doi:10.1080/00016480903143986

10. Rose TA, Choi JW. Intravenous imaging contrast media complications: the basics that every clinician needs to know. Am J Med (2015) 128:943-9. doi:10.1016/j.amjmed.2015.02.018

11. Parmar C, Leijenaar RTH, Grossmann P, Rios Velazquez E, Bussink J, Rietveld D, et al. Radiomic feature clusters and prognostic signatures specific for lung and head \& neck cancer. Sci Rep (2015) 5:11044. doi:10.1038/ srep11044

12. Aerts HJ, Velazquez ER, Leijenaar RT, Parmar C, Grossmann P, Carvalho S, et al. Decoding tumour phenotype by noninvasive imaging using a quantitative radiomics approach. Nat Commun (2014) 5:4006. doi:10.1038/ncomms5006

13. de Albuquerque M, Anjos LGV, Maia Tavares de Andrade H, de Oliveira MS, Castellano G, Junqueira Ribeiro de Rezende T, et al. MRI texture analysis reveals deep gray nuclei damage in amyotrophic lateral sclerosis. J Neuroimaging (2016) 26:201-6. doi:10.1111/jon.12262

14. Kumar V, Gu Y, Basu S, Berglund A, Eschrich SA, Schabath MB, et al. Radiomics: the process and the challenges. Magn Reson Imaging (2012) 30:1234-48. doi:10.1016/j.mri.2012.06.010

15. Lambin P, Rios-Velazquez E, Leijenaar R, Carvalho S, van Stiphout RG, Granton P, et al. Radiomics: extracting more information from medical images using advanced feature analysis. Eur J Cancer (2012) 48:441-6. doi:10.1016/j. ejca.2011.11.036 validating these results and translating them in a potential clinical diagnostic method to detect MD in MRI scans.

\section{AUTHOR CONTRIBUTIONS}

All the authors contributed to the design of the work presented in this paper. $\mathrm{EB}$ and $\mathrm{MH}$ designed the experiment, gathered the data, performed the analysis, and wrote the manuscript. RB designed the experiment, performed the analysis, supervised the writing, reviewed the manuscript, and edited the manuscript. AP and AJ performed the analysis, reviewed the manuscript, and edited the manuscript. RS and HK reviewed the manuscript and edited the manuscript. All the authors take full responsibility for the correctness of this paper and approved the final version.
16. Dang M, Lysack JT, Wu T, Matthews TW, Chandarana SP, Brockton NT, et al. MRI texture analysis predicts p53 status in head and neck squamous cell carcinoma. AJNR Am J Neuroradiol (2015) 36:166-70. doi:10.3174/ajnr.A4110

17. Coroller TP, Grossmann P, Hou Y, Rios Velazquez E, Leijenaar RT, Hermann G, et al. CT-based radiomic signature predicts distant metastasis in lung adenocarcinoma. Radiother Oncol (2015) 114:345-50. doi:10.1016/ j.radonc.2015.02.015

18. De Oliveira MS, Balthazar MLF, D'Abreu A, Yasuda CL, Damasceno BP, Cendes F, et al. MR imaging texture analysis of the corpus callosum and thalamus in amnestic mild cognitive impairment and mild Alzheimer disease. AJNR Am J Neuroradiol (2011) 32:60-6. doi:10.3174/ajnr.A2232

19. Slicer. $B W H$ and $3 D$ Slicer Contributors. (2015). Available from: http://www. slicer.org

20. Fedorov A, Beichel R, Kalpathy-Cramer J, Finet J, Fillion-Robin JC, Pujol S, et al. 3D Slicer as an image computing platform for the Quantitative Imaging Network. Magn Reson Imaging (2012) 30:1323-41. doi:10.1016/j. mri.2012.05.001

21. Pender DJ. Endolymphatic hydrops and Meniere's disease: a lesion meta-analysis. J Laryngol Otol (2014) 128:859-65. doi:10.1017/s0022215114001972

22. Kim SH, Kim JY, Lee HJ, Gi M, Kim BG, Choi JY. Autoimmunity as a candidate for the etiopathogenesis of Meniere's disease: detection of autoimmune reactions and diagnostic biomarker candidate. PLoS One (2014) 9:e111039. doi:10.1371/journal.pone.0111039

23. Yilmaz A, Ulak FS, Batun MS. Proton T1 and T2 relaxivities of serum proteins. Magn Reson Imaging (2004) 22:683-8. doi:10.1016/j.mri.2004.02.001

24. Parmar C, Grossmann P, Bussink J, Lambin P, Aerts HJ. Machine learning methods for Quantitative Radiomic Biomarkers. Sci Rep (2015) 5:13087. doi:10.1038/srep13087

25. Gurkov R, Berman A, Dietrich O, Flatz W, Jerin C, Krause E, et al. MR volumetric assessment of endolymphatic hydrops. Eur Radiol (2015) 25:585-95. doi:10.1007/s00330-014-3414-4

26. Pykkö I, Nakashima T, Yoshida T, Zou J, Naganawa S. Ménière’s disease: a reappraisal supported by a variable latency of symptoms and the MRI visualisation of endolymphatic hydrops. BMJ Open (2013) 3:e001555. doi:10.1136/ bmjopen-2012-001555

27. Barath K, Schuknecht B, Naldi AM, Schrepfer T, Bockisch CJ, Hegemann SC. Detection and grading of endolymphatic hydrops in Meniere disease using MR imaging. AJNR Am J Neuroradiol (2014) 35:1387-92. doi:10.3174/ajnr. A3856

28. Gurkov R, Flatz W, Louza J, Strupp M, Ertl-Wagner B, Krause E. In vivo visualized endolymphatic hydrops and inner ear functions in patients with electrocochleographically confirmed Meniere's disease. Otol Neurotol (2012) 33:1040-5. doi:10.1097/MAO.0b013e31825d9a95

29. Fiorino F, Pizzini FB, Beltramello A, Barbieri F. Progression of endolymphatic hydrops in Meniere's disease as evaluated by magnetic resonance imaging. Otol Neurotol (2011) 32:1152-7. doi:10.1097/MAO.0b013e318 22alce2

30. Gu X, Fang ZM, Liu Y, Huang ZW, Zhang R, Chen X. Diagnostic advantages of intratympanically gadolinium contrast-enhanced magnetic resonance 
imaging in patients with bilateral Meniere's disease. Am J Otolaryngol (2015) 36:67-73. doi:10.1016/j.amjoto.2014.10.003

Conflict of Interest Statement: The authors declare that the research was conducted in the absence of any commercial or financial relationships that could be construed as a potential conflict of interest.
Copyright (C) 2016 van den Burg, van Hoof, Postma, Janssen, Stokroos, Kingma and van de Berg. This is an open-access article distributed under the terms of the Creative Commons Attribution License (CC BY). The use, distribution or reproduction in other forums is permitted, provided the original author(s) or licensor are credited and that the original publication in this journal is cited, in accordance with accepted academic practice. No use, distribution or reproduction is permitted which does not comply with these terms. 